\title{
Comparison of tumor vascularity and hemodynamics in three rat hepatoma models
}

\author{
Jin Woo Choi, ${ }^{1}$ Jung Hoon Kim, ${ }^{1}$ Hyo-Cheol Kim, ${ }^{1}$ Won Seok Choi, ${ }^{1}$ Song Yi Baek, \\ Kyoungbun Lee, ${ }^{2}$ Jin Wook Chung ${ }^{1}$
}

${ }^{1}$ Department of Radiology, Seoul National University Hospital, Seoul National University College of Medicine, 101 Daehak-ro, Jongno-gu, Seoul 110-744, Republic of Korea

${ }^{2}$ Department of Pathology, Seoul National University Hospital, Seoul National University College of Medicine, Seoul, Republic of Korea

\begin{abstract}
Purpose: To compare tumor vascularity and hemodynamics in three rat hepatoma models: N1-S1 cells in Sprague-Dawley rats, McA-RH7777 cells in SpragueDawley rats, and 13762 MAT B III cells in F344 rats. Methods: The three rat hepatoma models were induced in five rats per group. After confirming that the tumors grew up to $10 \mathrm{~mm}$ on magnetic resonance imaging, the rats underwent dynamic contrast-enhanced ultrasonography (DCE-US). Afterward, the rats were euthanized for histologic analyses. The Kruskal-Wallis test was used to compare the rat hepatoma models. Correlation coefficients were calculated between the microvessel density (MVD) and DCE-US parameters.

Results: On DCE-US imaging, arterial enhancement and washout were demonstrated in all N1-S1 tumors, while persistent peripheral enhancement on arterial to portal phases was shown in all 13762 MAT B III tumors. The McA-RH7777 tumors presented diverse enhancement patterns on arterial and portal phases. There were no significant differences in DCE-US parameters among the three hepatoma groups, while MVD was correlated with peak intensity $(r=0.565, p=0.044)$, mean transit time $(r=-0.559, p=0.047)$, and time to peak $(r=-0.617$, $p=0.025)$ of individual rats. The necrosis ratio was significantly different between the models $(p=0.031)$; 13762 MAT B III showed a significantly higher necrosis ratio than N1-S1 ( $p<0.050$ by post hoc test).

Conclusion: The N1-S1 tumor may be suitable as a model to investigate hypervascular hepatic tumors of the liver in
\end{abstract}

Jin Woo Choi and Jung Hoon Kim have contributed equally to this work.

Correspondence to: Hyo-Cheol Kim; email: angiointervention@, gmail.com
DCE-US such as hepatocellular carcinoma among the three tumors.

Key words: Hepatocellular carcinoma-Rat hepatoma-Animal model-Vascularity-Contrastenhanced ultrasonography

Primary liver cancer is the sixth most prevalent cancer in the world, and the third most common cause of cancer mortality $[1,2]$. In particular, hepatocellular carcinoma (HCC) is a major health problem in Asia and Africa, and the incidence is rapidly increasing in Western countries [3-5]. Hence, a number of studies are being conducted on liver cancers in the pre-clinical research field.

Useful liver cancer models in experimental animals are required to translate laboratory studies to clinical applications. Rat hepatoma models are developed and used for animal research [6-9], in addition to conventional hepatoma models in mice and rabbits, because the rat models have the advantages of both of those species. Like mice, rats are applicable in diverse molecular and genetic experiments, and are easy to handle and breed compared to larger animals [10]. On the other hand, rats are large enough for applying complicated surgery and interventional treatments [11-13], and for studying novel imaging modalities [7, 14, 15]. For these reasons, diverse rat hepatoma models have recently been established for use in translational research $[8,16]$.

In this context, investigators who study liver cancer imaging or interventions should carefully choose a rat hepatoma model appropriate to their study aims. Although a recent study compared two rat hepatoma models (N1-S1 and McA-RH7777 cells in SpragueDawley rats), in terms of tumor growth pattern and 
immunologic reaction [17], until now, there has been very limited information about other characteristics among the rat tumor models. Considering that hypervascularity is a major feature of human HCC, which accounts for $70 \%-85 \%$ of primary liver cancers in the world [2], and that most liver imaging and intervention studies target this feature, knowledge of tumor vascularity is critical in deciding which hepatoma model will be selected.

Therefore, we conducted an animal study to compare tumor vascularity in three rat hepatoma models, N1-S1 cells in Sprague-Dawley rats, McA-RH7777 cells in Sprague-Dawley rats, and 13762 MAT B III cells in F344 rats, using dynamic contrast-enhanced ultrasonography (DCE-US) and immunohistochemistry.

\section{Materials and methods}

\section{Tumor cell line}

The N1-S1 (CRL-1604; ATCC, Manassas, VA, USA), McA-RH7777 (CRL-1601; ATCC), and 13762-MAT-BIII (CRL-1666; ATCC) rat tumor cell lines were obtained and cultured in RPMI-1640 (WelGENE, Daegu, Korea), Dulbecco's Modified Eagle's (WelGENE), and RPMI1640 media, respectively. The media were supplemented with 10\% fetal bovine serum (WelGENE) and a 1\% penicillin/streptomycin mixture (Gibco, Grand Island, NY, USA). The viability of the cells was tested with Trypan blue staining, which confirmed $>90 \%$ cell viability for tumor implantation procedures.

\section{Animal modeling}

This study was approved by our institutional animal care and use committee, and it was performed in accordance with institutional guidelines. Ten male Sprague-Dawley rats initially weighing about $400 \mathrm{~g}$ and five F344 rats weighing about $300 \mathrm{~g}$ were used for this study. A mixture of zolazepam ( $5 \mathrm{mg} / \mathrm{kg}$, Zoletil ${ }^{\circledR}$; Virbac, Carros, France) and xylazine $\left(10 \mathrm{mg} / \mathrm{kg}\right.$, Rompun $^{\circledR}$; Bayer-Schering Pharma, Berlin, Germany) was intramuscularly injected into the hindlimb to anesthetize each rat. Next, a minilaparotomy was conducted to expose the left lateral lobe of the liver. According to the established protocols for each tumor model $[12,16-18]$, the N1-S1 $\left(5 \times 10^{6}\right.$ cells prepared in $50 \mu \mathrm{L}$ of medium), McA-RH7777 $\left(5 \times 10^{6}\right.$ cells in $50 \mu \mathrm{L})$, and 13762 MAT B III $\left(1 \times 10^{5}\right.$ cells in $50 \mu \mathrm{L})$ cell lines were injected gently under the hepatic capsule of five Sprague-Dawley rats, five SpragueDawley rats, and five F344 rats, respectively. Handheld electrocautery (Bovie Medical Corporation, Clearwater, FL, USA) was applied to prevent tumor cell reflux and to stop the bleeding, and a 2-layer abdominal incision closure was subsequently performed. To prevent spontaneous tumor regression of the N1-S1 and McARH7777 hepatomas, cyclosporine A $(20 \mathrm{mg} / \mathrm{kg} / \mathrm{day}$; Chong Kun Dang Pharmaceutical Corp., Seoul, Korea) was subcutaneously administrated from 1 day before the tumor implantation until 4 days after the surgery [9].

\section{Tumor growth monitoring}

From the seventh day after each cell line injection, tumor induction and growth were monitored every third day with a 3.0-Tesla magnetic resonance scanner (TrioTim; Siemens Medical Solutions, Erlangen, Germany) with a six-channel rat-body coil (Stark Contrast, Erlangen, Germany). Axial T2-weighted images (repetition time/ echo time $=3,800 / 78 \mathrm{~ms}$; bandwidth $=199 \mathrm{~Hz} /$ pixel; flip angle $=140^{\circ}$; slice thickness $=2 \mathrm{~mm}$; field of view $=120 \times 109 \mathrm{~mm}$; matrix $=256 \times 197$ ) were obtained for each animal. After confirming tumor growth of up to $10 \mathrm{~mm}$ on magnetic resonance imaging, the rats underwent subsequent DCE-US studies.

\section{Dynamic contrast-enhanced ultrasonography}

DCE-US scans were conducted 1 day after the magnetic resonance scans by an abdominal radiologist (J.H.K) who was blinded to the type of tumor implanted in each rat [19-21]. After achieving anesthesia by the same method used for tumor implantation, each rat bearing a hepatoma was manually injected with $0.1 \mathrm{~mL}$ of the contrast agent, sulfur hexafluoride-filled microbubbles (SonoVue; Bracco, Milan, Italy), followed by $0.5 \mathrm{~mL}$ of a normal saline flush through the tail vein [20]. DCE-US images were acquired with a PLT-120 equipped with a $12-\mathrm{MHz}$ center-frequency linear transducer using the following parameters: a dynamic range of 65 , a mechanical index of 0.09 , a gain of 95 , and a depth of $1 \mathrm{~cm}$, equipped in an ultrasound scanner (Aplio500; Toshiba Medical Systems, Otawara, Japan). A fundamental B-mode scan was conducted to identify the hepatomas and to measure the longest diameter $(\mathrm{mm})$ of each tumor. The DCE-US data were obtained by the contrast harmonic image mode (CHI, Toshiba Medical Systems, Japan) with a low mechanical index of 0.09 , which can detect the signal generated by microbubbles. The images were continuously recorded beginning at the time of the contrast agent injection and for 90 additional seconds (eight frames per second).

\section{Imaging analysis}

Based on the DCE-US images, tumor enhancement patterns (echogeneity on arterial/portal/delayed phases, and homogeneous/heterogeneous/peripheral enhancement on arterial phase) were qualitatively characterized by two independent radiologists (J.W.C and W.S.C) who were blinded to the type of tumor implanted in each rat. Subjectively, the portal phase was defined as the duration when the normal liver parenchyma was strongly and homogeneously enhanced. The arterial and delayed phases were determined as the times before and after the 
portal phase, respectively. After the initial, independent review of the DCE-US images, interobserver agreement $(\kappa)$ for the assessment of the tumor enhancement patterns was calculated. Discordant results were then resolved by consensus review of the two radiologists.

The quantitative parameters of DCE-US were analyzed using the dedicated software CHI-Q (Toshiba Medical Systems). A region of interest (ROI) along the margin of each tumor at a selected frame was drawn manually, and the ROI was auto-positioned throughout all of the images. If there were changes in the tumor position due to respiratory motion during the scanning, the ROI at a misregistered frame was adjusted manually and the software automatically interpolated the ROIs between well-registered and misregistered frames. Based on the time-intensity curves of the ROIs, the peak intensity, slope coefficient of the wash-in $\left(\mathrm{SC}_{\text {in }}\right)$, time to peak intensity (TTP), mean transit time (MTT), total area under the time-intensity curve $\left(\mathrm{AUC}_{\text {total }}\right), \mathrm{AUC}$ during the wash-in $\left(\mathrm{AUC}_{\mathrm{in}}\right)$, and $\mathrm{AUC}$ during the washout $\left(\mathrm{AUC}_{\text {out }}\right)$ were calculated automatically.

\section{Histologic analysis}

The rats were euthanized in a $\mathrm{CO}_{2}$ chamber within $24 \mathrm{~h}$ after DCE-US study, and the tumors, including adjacent liver parenchyma, were harvested. Axial sections sampled across the center of the tumors were fixed in a $10 \%$ buffered formaldehyde solution and embedded in paraffin. The slides were stained with hematoxylin and eosin and CD34 (Rat CD34 antibody; R\&D Systems, Minneapolis, MN, USA), and then digitalized with optical magnification $(\times 200)$ to conduct further analyses.

Tumor morphology and cytological features were assessed on the hematoxylin and eosin slides. The necrosis ratio (\%) was defined as the ratio of necrotic area drawn manually on the digitalized slides per total tumor area, which was evaluated using software (ImageJ, version 1.48; National Institutes of Health, USA). One investigator (S.Y.B) who was blinded to the entire study design selected three hot spots per rat on a CD34 slide with optical magnification $\left(\times 200,0.22 \mathrm{~mm}^{2}\right)$. The hot spots corresponded to the area that showed the highest density of CD34 expression without non-specific uptake in the given samples. On the hot spot images, the number of microvessels apart from each other and with identifiable intravascular lumen was counted, and the mean value of three measurements was calculated as the MVD [22]. The CD34 expression in the capillarized sinusoids, if any, was separately recorded, but was not considered in the calculation of MVD because of its nest-like, uncountable features [23].

\section{Statistical analysis}

Interobserver agreement about the tumor enhancement patterns on DCE-US images was expressed with the
Cohen $\kappa$ coefficient. A $\kappa$ statistic of $0.80-1.00$ was considered indicative of excellent agreement; $0.60-0.79$, good agreement; $0.40-0.59$, moderate agreement; $0.20-0.39$, fair agreement; and $0-0.19$, poor agreement. The Kruskal-Wallis test was used to compare tumor size measured on ultrasonography, DCE-US parameters (peak intensity, $\mathrm{SC}_{\text {in }}, \mathrm{TTP}, \mathrm{MTT}, \mathrm{AUC}_{\text {total }}, \mathrm{AUC}_{\mathrm{in}}$, and $\mathrm{AUC}_{\text {out }}$ ), MVD, and necrosis ratio among the tumor models (post hoc test by Conover's method). Pearson's correlation coefficient $(r)$ was calculated to evaluate the relationship of MVD to DCE-US parameters. A $p$ value of $<0.050$ was considered to be statistically significant, and statistical analyses were performed using commercial software $\left(\right.$ MedCalc $^{\circledR}$, version 14.12.0; MedCalc Software, Ostend, Belgium).

\section{Results}

\section{Tumor modeling}

Thirteen out of 15 rats were evaluated according to our protocols. Two rats (one in the N1-S1 group and one in the 13762 MAT B III group) that expired during the anesthesia were excluded for analysis. The N1-S1 $(n=4)$, McA-RH-7777 $(n=5)$, and 13762 MAT B III $(n=4)$ tumors reached about $10 \mathrm{~mm}$ in diameter on $10-$, 16-, and 16-day magnetic resonance images, respectively. The mean \pm standard deviation $(\mathrm{SD})$ values of the corresponding tumor groups were $14.0 \pm 1.2,12.0 \pm 3.6$, and $11.7 \pm 1.3 \mathrm{~mm}$, respectively. The tumor sizes were not significantly different at the time of the vascularity evaluation $(p=0.285)$.

\section{Tumor characteristics}

All tumors were identified on B-mode ultrasonography as heterogeneously hyperechoic lesions compared to the liver parenchyma. Tumor enhancement patterns on DCE-US were different depending on the type of tumor (Table 1). Arterial enhancement and washout on portal/ delayed phases were demonstrated in all N1-S1 tumors, while persistent peripheral enhancement on arterial to portal phases was shown in all of the 13762 MAT B III tumors (Fig. 1). The McA-RH7777 tumors presented diverse enhancement patterns on arterial and portal phases. In terms of DCE-US parameters, there were no significant differences in the values among each hepatoma group. Interobserver agreement about the assessment of the tumor enhancement patterns was good $(\kappa=0.784)$.

The hematoxylin and eosin staining demonstrated poorly differentiated cell proliferation in the N1-S1 and 13762 MAT B III tumors, in contrast to well to moderately differentiated cell proliferation with preserved hepatic trabecular architectures in the McA-RH7777 tumors (Fig. 2). The MVD was not significantly different between the three groups $(p=0.175)$, while the McA- 
Table 1. Characteristics of three rat hepatoma models

\begin{tabular}{|c|c|c|c|c|}
\hline & \multicolumn{4}{|c|}{ Cell line } \\
\hline & $\begin{array}{c}\text { N1-S1 } \\
(n=4)\end{array}$ & $\begin{array}{c}\text { McA-RH7777 } \\
(n=5)\end{array}$ & $\begin{array}{l}\text { MAT-B-III-13762 } \\
(n=4)\end{array}$ & $P$ value \\
\hline Tumor size $(\mathrm{mm})^{\mathrm{a}}$ & $14.0 \pm 1.2$ & $12.0 \pm 3.6$ & $11.7 \pm 1.3$ & 0.285 \\
\hline \multicolumn{5}{|l|}{ DCE-US imaging } \\
\hline Hyper-/hypoechoic on AP & $4 / 0$ & $2 / 3$ & $4 / 0$ & \multirow[t]{4}{*}{$\mathrm{NE}$} \\
\hline Hyper-/hypoechoic on PP & $0 / 4$ & $3 / 2$ & $4 / 0$ & \\
\hline Hyper-/hypoechoic in DP & $0 / 4$ & $0 / 5$ & $2 / 2$ & \\
\hline Homo/Hete/Peri enhancement ${ }^{b}$ & $2 / 2 / 0$ & $3 / 1 / 1$ & $0 / 0 / 4$ & \\
\hline \multicolumn{5}{|l|}{ DCE-US parameter ${ }^{\mathrm{a}}$} \\
\hline Peak intensity (AU) & $7.4 \pm 4.7$ & $4.9 \pm 3.6$ & $6.5 \pm 3.1$ & 0.526 \\
\hline $\mathrm{SC}_{\text {in }}(\mathrm{AU} / \mathrm{s})$ & $1.9 \pm 1.5$ & $1.9 \pm 2.1$ & $3.2 \pm 1.7$ & 0.242 \\
\hline TTP (s) & $5.6 \pm 1.3$ & $6.1 \pm 3.1$ & $3.1 \pm 1.1$ & 0.088 \\
\hline MTT (s) & $15.7 \pm 2.8$ & $27.3 \pm 26.0$ & $11.4 \pm 5.1$ & 0.248 \\
\hline $\mathrm{AUC}_{\text {total }}(\mathrm{AU} \cdot \mathrm{s})$ & $230.2 \pm 148.2$ & $151.8 \pm 58.3$ & $131.8 \pm 75.3$ & 0.458 \\
\hline $\mathrm{AUC}_{\text {in }}(\mathrm{AU} \cdot \mathrm{s})$ & $26.5 \pm 14.9$ & $14.9 \pm 6.9$ & $13.8 \pm 8.6$ & 0.206 \\
\hline $\mathrm{AUC}_{\text {out }}(\mathrm{AU} \cdot \mathrm{s})$ & $203.8 \pm 133.7$ & $136.9 \pm 52.2$ & $118.0 \pm 70.0$ & 0.371 \\
\hline \multicolumn{5}{|l|}{ Histologic finding ${ }^{\mathrm{a}}$} \\
\hline MVD (microvessel $/ 0.22 \mathrm{~mm}^{2}$ ) & $21.6 \pm 5.1$ & $19.1 \pm 18.9$ & $36.9 \pm 7.7$ & 0.175 \\
\hline Necrosis ratio $(\%)$ & $22.4 \pm 8.8$ & $34.2 \pm 9.7$ & $45.6 \pm 8.4$ & 0.031 \\
\hline
\end{tabular}

$A P$ arterial phase, $P P$ portal phase, $D P$ delayed phase, Homo homogeneous, Hete heterogeneous, Peri peripheral, $A U$ arbitrary unit, $S C_{i n}$ slope coefficient of the wash-in, $T T P$ time to peak intensity, $M T T$ mean transit time, $A U C_{\text {total }}$ total area under the time-intensity curve, $A U C_{i n}$ AUC during the wash-in, $A U C_{\text {out }}$ AUC during the wash-out, $M V D$ microvessel density, $N E$ not evaluated

${ }^{a}$ Data are mean \pm standard deviation

b The tumor enhancement pattern was assessed on the arterial phase images

RH7777 group demonstrated substantial in-group variability of MVD (mean \pm SD, $19.1 \pm 18.9$ microvessels/ $0.22 \mathrm{~mm}^{2}$ ) (Table 1). Capillarization of the sinusoids was detected exclusively in the McA-RH7777 group. The necrosis ratio was significantly different between the three groups $(p=0.031)$, and post hoc test revealed that the 13762 MAT B III tumors showed significantly higher necrosis ratios than those of the N1-S1 tumors ( $p<0.050$ by Conover's method) (Table 1; Fig. 3).

\section{Correlation between image findings and microvessel density}

The MVD of the 13 rats was positively correlated with peak intensity $(r=0.565, p=0.044)$, and negatively correlated with MTT $(r=-0.559, p=0.047)$ and TTP $(r=-0.617, p=0.025)$, among the DCE-US parameters (Fig. 4).

\section{Discussion}

This study revealed that the three rat hepatoma models are conspicuously different in terms of tumor vascularity, necrosis propensity, and cytologic features. Vascularity is regarded as a key feature in characterizing a tumor, and HCC is well known to have remarkably increased vascularity due to neoangiogenesis. As unpaired arteries are increased, HCC receives arterial blood flow predominantly, which results in early enhancement on arterial phase and washout in the portal/delayed phase in dynamic imaging studies [24, 25]. In addition, the hyper- vascularity of $\mathrm{HCC}$ is targeted in diverse treatments, including transarterial chemoembolization and sorafenib $[24,25]$. Therefore, tumor vascularity should be meticulously considered by every investigator who studies liver cancer.

In this study, the N1-S1 tumors demonstrated the typical enhancement patterns of human HCC (arterial enhancement followed by washout on portal/delayed phase) on qualitatively assessed DCE-US findings. The peak intensity, $\mathrm{AUC}_{\text {total }}, \mathrm{AUC}_{\mathrm{in}}$, and $\mathrm{AUC}_{\text {out }}$ were highest in the N1-S1 group, although statistical significance was not proved in our study. Theoretically, those values can increase when a tumor is hypervascular and unlikely to have necrosis. Histologic findings confirmed proliferation of poorly differentiated cells with the lowest propensity for necrosis compared to the other hepatoma models. Considering these features, the N1-S1 model may be the most appropriate model to study the imaging-based diagnosis and treatment of HCC.

On the other hand, the McA-RH7777 tumors presented high variability of imaging findings as well as of histologic characteristics, while the hepatic trabecular architecture was relatively preserved. Of note, capillarization of sinusoids, which is a distinctive feature of the cirrhotic liver and HCC, was found in this tumor model [23]. Therefore, the McA-RH7777 model may be suitable for studies on the tumor heterogeneity and pathogenesis of HCC.

The 13762 MAT B III model, a metastatic hepatoma model, showed persistent peripheral enhancement on arterial to portal phases of DCE-US scan, which mir- 

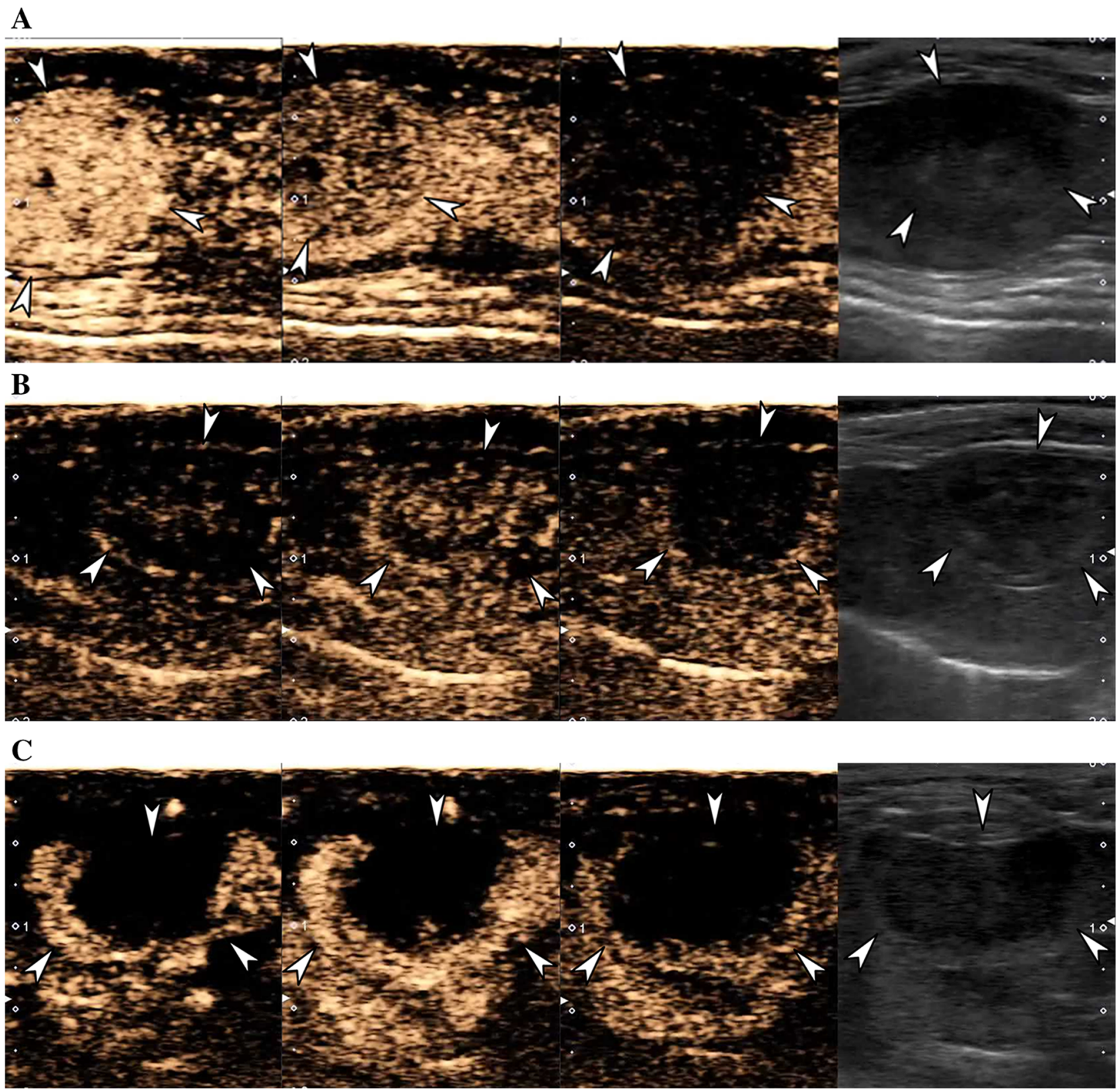

Fig. 1. Dynamic contrast-enhanced ultrasonography imaging of three rat hepatoma models (from left to right: arterial phase, portal phase, delayed phase, and B-mode imaging). A N1-S1 tumor (arrowheads) demonstrating homogeneous arterial enhancement and washout in the portal and delayed phases. B McA-RH7777 tumor (arrowheads) demonstrating

rored extensive central necrosis (necrosis ratio, $45.6 \%$ ). This phenomenon may explain the lower peak intensity, $\mathrm{AUC}_{\text {total }}, \mathrm{AUC}_{\mathrm{in}}$, and $\mathrm{AUC}_{\mathrm{out}}$ values compared to that of the N1-S1 model, in spite of the highest MVD $\left(36.9 \pm 7.7\right.$ microvessels $\left./ 0.22 \mathrm{~mm}^{2}\right)$ of the $13762 \mathrm{MAT}$ B III model, measured in viable tumor portions. Because the aforementioned DCE-US parameters reflect the no definite enhancement in the arterial phase, heterogeneous enhancement in the portal phase, and washout in the delayed phase. C 13762 MAT B III tumor (arrowheads) demonstrating persistent peripheral enhancement in the arterial, portal, and delayed phases.

sum of signal intensity changes in the total tumor area scanned, massive necrosis (i.e., non-enhancing areas) deteriorates a degree of alteration. Concordantly, extensive central necrosis can frequently be seen in metastatic liver tumors [26]. Hence, this model can be a useful tool for investigators studying liver metastases of hypervascular tumors. 

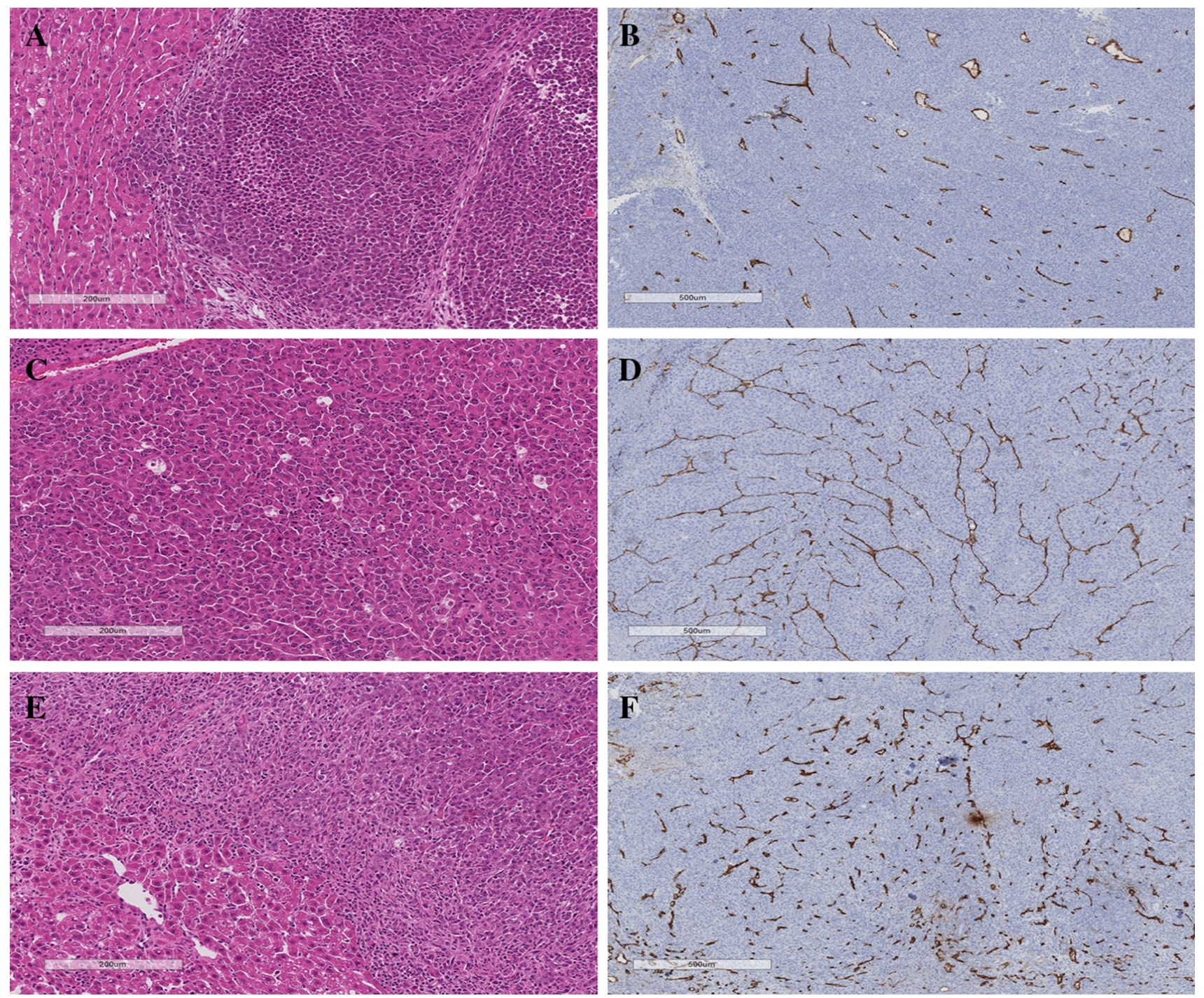

Fig. 2. Histologic features of three rat hepatoma models. A N1-S1 tumor showing proliferation of poorly differentiated cells (hematoxylin and eosin, $\times 100$ magnification). B N1-S1 tumor showing multiple distinct microvessels (CD34, $\times 40$ magnification). C McA-RH7777 tumor showing cell proliferation with relatively preserved hepatic trabecular architecture

In spite of the conspicuous differences in histologic features, the MVD and DCE-US parameters were not significantly different among the tumor groups. On the other hand, the MVDs of individual rats were reasonably correlated with peak intensity, MTT, and TTP on the DCE-US scan. A higher MVD value was significantly correlated to the faster enhancement (i.e., smaller TTP) and washout (i.e., smaller MTT) of a tumor [27]. A higher peak intensity value, related to higher MVD, was also associated with fast influx and washout of microbubbles, assuming the amount of microbubbles passing through a tumor is consistent [27]. These findings suggested that the DCE-US parameters were reli-

(hematoxylin and eosin, $\times 100$ magnification). D McA-RH7777 tumor showing prominent capillarization of the sinusoids (CD34, $\times 40$ magnification). E 13762 MAT B III tumor showing proliferation of poorly differentiated cells (hematoxylin and eosin, $\times 100$ magnification). F 13762 MAT B III tumor showing numerous distinct microvessels (CD34, $\times 40$ magnification).

able, but in-group variability of tumor vascularity was considerable.

In particular, the McA-RH7777 tumors demonstrated the largest variability in terms of MVD, necrosis ratio, $\mathrm{SC}_{\mathrm{in}}$, TTP, MTT, and tumor size during the same incubation period (see SD of the variables in Table 1). Various degrees of immune reaction in each rat can affect the heterogeneous vascularity of McA-RH7777 tumors in our study [17], considering that this cell line does not originate from Sprague-Dawley rats but from Buffalo rats. Capillarization of sinusoids in the McA-RH7777 model may also contribute to the calculation of MVD and DCE-US parameters. Meanwhile, Guo et al. [18] 


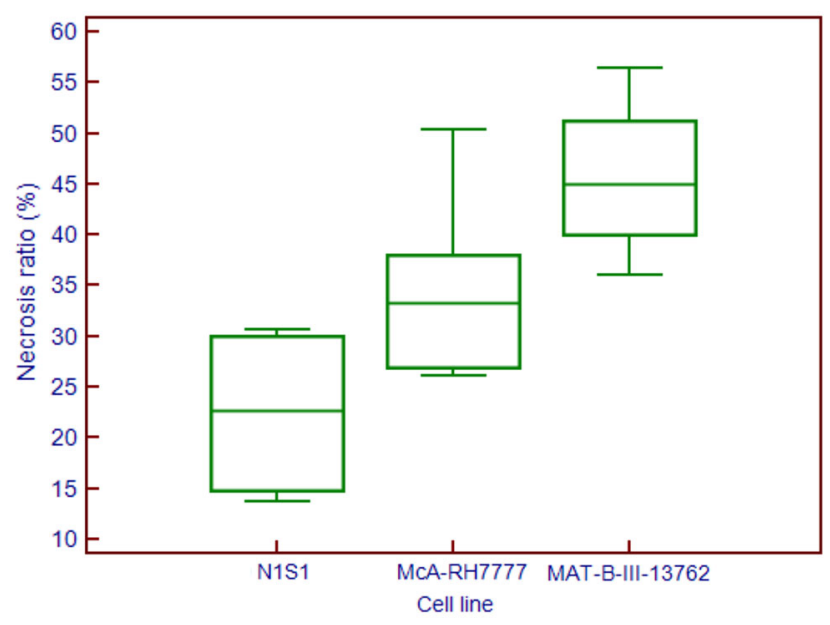

Fig. 3. Box-and-whisker plot representing the different necrosis ratios of the three rat hepatoma models.

claimed that McA-RH7777 tumors presented a higher MVD than N1-S1 tumors, showing prominent CD34 uptake in the sinusoidal-like vessels. That result is quite different from our MVD analysis, which may be due to the fact that Guo et al. [18] digitally evaluated CD34-positive pixels per total tumor pixels while we visually counted microvessels apart from each other and having identifiable vascular lumen, in order to rule out capillarized sinusoids. The CD34 slides in our study concordantly demonstrated a substantial portion of CD34-positive areas but relatively sparse microvessels distinctly separated from each other (Fig. 2D).

There are a few limitations in our study. Firstly, the microbubble contrast agent was manually injected into each rat. Although the injection was performed by a blinded and well-trained radiographer, this may affect the in-group variability of the DCE-US parameters. Secondly, the N1-S1 and McA-RH7777 tumors were induced by means of an immunosuppressant (cyclosporine A) [9]. Considering the spontaneous tumor regression propensity of these models without immune suppression [17], the serial changes of vascularity depending on tumor-incubation periods, and the immunosuppressant effects on tumor vascularity need to be evaluated. Thirdly, the number of animals studied was limited. Considering the in-group variability of our experiment, further evaluation with a larger study population may show more distinguishable results.

In conclusion, the N1-S1 tumor may be suitable as a model to investigate hypervascular hepatic tumors of the liver in DCE-US such as hepatocellular carcinoma among the three tumors.
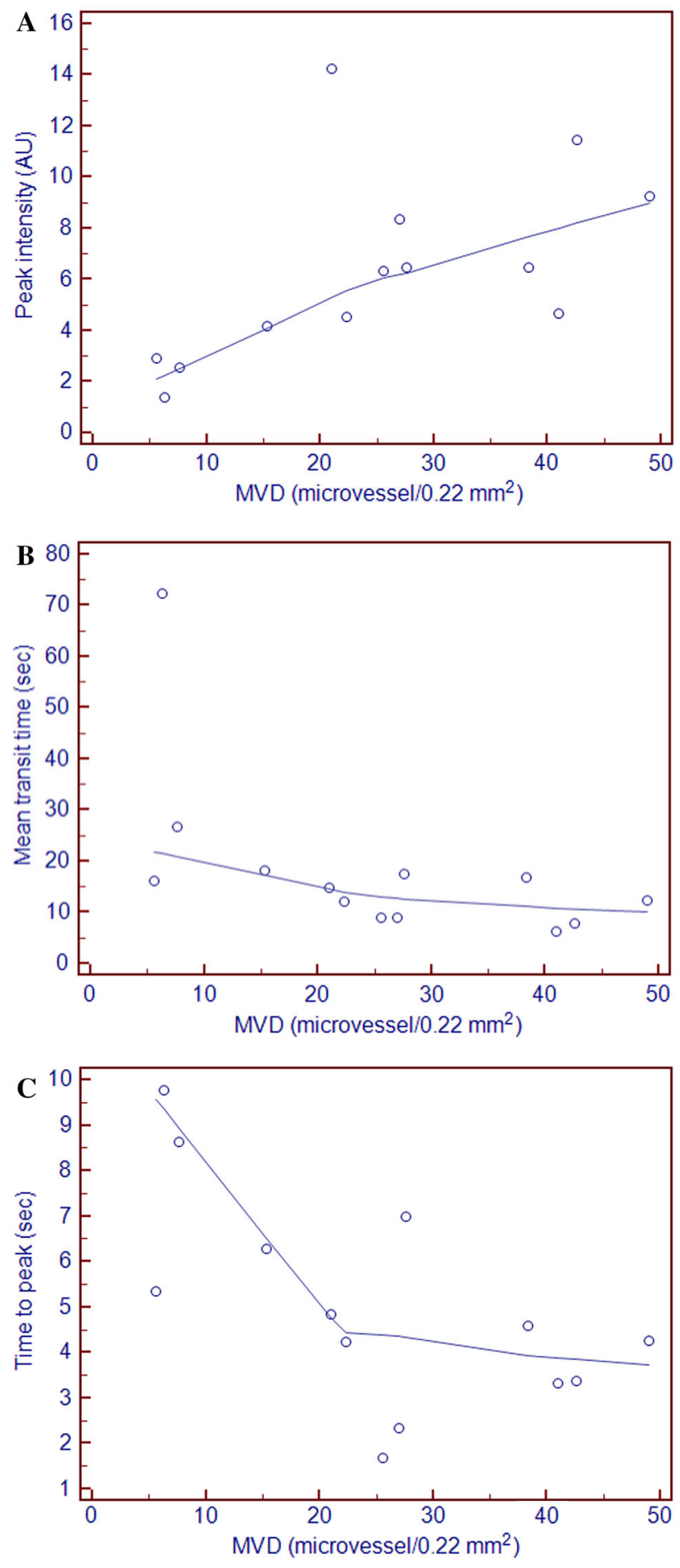

Fig. 4. Correlation of microvessel density (MVD) to dynamic contrast-enhanced ultrasonography parameters. A Peak intensity (presented as arbitrary unit, AU) is positively correlated with MVD $(r=0.565, p=0.044)$. B Mean transit time is negatively correlated with MVD $(r=-0.559, p=0.047)$. C Time to peak is negatively correlated with MVD $(r=-0.617$, $p=0.025)$. 
Acknowledgments. This study was supported by the SNUH Research Fund (04-2013-0790) and by the National Research Foundation of Korea (NRF-2013R1A1A2A10011007) from the government of South Korea.

\section{Compliance with Ethical Standards}

Conflict of interest The authors declare that they have no conflict of interest.

\section{References}

1. McGlynn KA, London WT (2011) The global epidemiology of hepatocellular carcinoma: present and future. Clin Liver Dis 15(2):223-243

2. Jemal A, Bray F, Center MM, et al. (2011) Global cancer statistics. CA Cancer J Clin 61(2):69-90

3. Nordenstedt H, White DL, El-Serag HB (2010) The changing pattern of epidemiology in hepatocellular carcinoma. Dig Liver Dis 42(Suppl 3):S206-S214

4. Center MM, Jemal A (2011) International trends in liver cancer incidence rates. Cancer Epidemiol Biomarkers Prev 20(11):2362-2368

5. El-Serag HB, Kanwal F (2014) Epidemiology of hepatocellular carcinoma in the United States: where are we? Where do we go? Hepatology 60(5):1767-1775

6. Gordon AC, Lewandowski RJ, Salem R, et al. (2014) Localized hyperthermia with iron oxide-doped yttrium microparticles: steps toward image-guided thermoradiotherapy in liver cancer. J Vasc Interv Radiol 25(3):397-404

7. Choi JW, Kim H, Kim HC, et al. (2013) Blood oxygen level-dependent MRI for evaluation of early response of liver tumors to chemoembolization: an animal study. Anticancer Res 33(5):1887-1892

8. Thompson SM, Callstrom MR, Knudsen B, et al. (2013) AS30D model of hepatocellular carcinoma: tumorigenicity and preliminary characterization by imaging, histopathology, and immunohistochemistry. Cardiovasc Intervent Radiol 36(1):198-203

9. Lee TK, Na KS, Kim J, Jeong HJ (2014) Establishment of animal models with orthotopic hepatocellular carcinoma. Nucl Med Mol Imaging 48(3):173-179

10. Suda T, Liu D (2007) Hydrodynamic gene delivery: its principles and applications. Mol Ther 15(12):2063-2069

11. Du Z, Wei C, Cheng K, et al. (2013) Mesenchymal stem cell-conditioned medium reduces liver injury and enhances regeneration in reduced-size rat liver transplantation. J Surg Res 183(2):907-915

12. Cho HR, Choi JW, Kim HC, et al. (2013) Sprague-Dawley rats bearing McA-RH7777 cells for study of hepatoma and transarterial chemoembolization. Anticancer Res 33(1):223-230
13. Mouli SK, Tyler P, McDevitt JL, et al. (2013) Image-guided local delivery strategies enhance therapeutic nanoparticle uptake in solid tumors. ACS Nano 7(9):7724-7733

14. Guo Y, Jin N, Klein R, et al. (2012) Gas challenge-blood oxygen level-dependent (GC-BOLD) MRI in the rat Novikoff hepatoma model. Magn Reson Imaging 30(1):133-138

15. Wang YX, Yuan J, Chu ES, et al. (2011) T1rho MR imaging is sensitive to evaluate liver fibrosis: an experimental study in a rat biliary duct ligation model. Radiology 259(3):712-719

16. Choi JW, Kim HC, Baek SY, Ryu YJ, Chung JW (2015) A metastatic hepatoma model of rats using the 13762-MAT-B-III cell line: basic characteristics and potential as a tool for interventional oncology experiments. Anticancer Res 35(3):1333-1338

17. Buijs M, Geschwind JF, Syed LH, et al. (2012) Spontaneous tumor regression in a syngeneic rat model of liver cancer: implications for survival studies. J Vasc Interv Radiol 23(12):1685-1691

18. Guo Y, Klein R, Omary RA, Yang GY, Larson AC (2010) Highly malignant intra-hepatic metastatic hepatocellular carcinoma in rats. Am J Transl Res 3(1):114-120

19. Bokor D (2000) Diagnostic efficacy of SonoVue. Am J Cardiol $86(4 \mathrm{~A}): 19 \mathrm{G}-24 \mathrm{G}$

20. Joo I, Kim JH, Lee JM, et al. (2014) Early quantification of the therapeutic efficacy of the vascular disrupting agent, CKD-516, using dynamic contrast-enhanced ultrasonography in rabbit VX2 liver tumors. Ultrasonography 33(1):18-25

21. Lassau N, Koscielny S, Chami L, et al. (2011) Advanced hepatocellular carcinoma: early evaluation of response to bevacizumab therapy at dynamic contrast-enhanced US with quantificationpreliminary results. Radiology 258(1):291-300

22. Kim HC, Chung JW, Choi SH, et al. (2012) Augmentation of chemotherapeutic infusion effect by TSU-68, an oral targeted antiangiogenic agent, in a rabbit VX2 liver tumor model. Cardiovasc Intervent Radiol 35(1):168-175

23. Tanigawa N, Lu C, Mitsui T, Miura S (1997) Quantitation of sinusoid-like vessels in hepatocellular carcinoma: its clinical and prognostic significance. Hepatology 26(5):1216-1223

24. Bruix J, Sherman M, American Association for the Study of Liver Diseases (2011) Management of hepatocellular carcinoma: an update. Hepatology 53(3):1020-1022

25. European Association For The Study Of The Liver, European Organisation For Research and Treatment Of Cancer (2012) EASL-EORTC clinical practice guidelines: management of hepatocellular carcinoma. J Hepatol 56(4):908-943

26. Sica GT, Ji H, Ros PR (2000) CT and MR imaging of hepatic metastases. AJR Am J Roentgenol 174(3):691-698

27. Kong WT, Yuan HX, Cai H, et al. (2015) Early treatment response to sorafenib for rabbit VX2 orthotic liver tumors: evaluation by quantitative contrast-enhanced ultrasound. Tumour Biol 36(4): 2593-2599 